http://dx.doi.org/10.21611/qirt.1994.043

\title{
Lock-in infrared thermography applied to the characterization of electromagnetic fields
}

by NACITAS M., LEVESQUE P. and BALAGEAS D.

ONERA , L3C, BP 72 F-92322 Châtillon-Cedex, France

\begin{abstract}
Lock-in infrared thermographic systems developed since more than ten years allow the measurement of both amplitude and phase of periodic temperature fields. They have been essentially used for mechanical testing, mainly for stress field analysis, and for NDE. The present system is applied to the quantitative characterization of electromagnetic fields. The modulated amplitude of the temperature leads to the intensity of the field. The purpose of such a system is to eliminate both radiato-convective and conductive effects which produce a blurring of the thermal image and hence degrade the accuracy of the measurement. Experiments demonstrate the real enhancement of the technique when compared to classical steady state measurements. The interest of the thermal phase image is also presented.
\end{abstract}

\section{Nomenclature}

$h$ heat transfer coefficient for losses

$\omega$ modulation pulsation

$C$ volume specific heat of a material

$f$ modulation frequency

\author{
$e$ thickness of the film \\ $\delta$ diffusion length \\ $\kappa$ thermal diffusivity of a material
}

\section{Introduction}

Infrared (IR) thermography is used since several years as an imaging tool for mapping microwave fields in free field conditions or in cavities, surface current and surface charge distributions on conducting structures, or more recently, energy absorption in microwave absorbing materials [1-2]. The heat produced by the electromagnetic (EM) waves absorption by various materials and structures is imaged with the following advantages : non contact measurements, fast operations, possible reversibility of the phenomenon, easy handling of data.

The evolution of the IR thermographic equipment and techniques allows quantitative measurements that a few researchers have tried to really obtain in this field of application [3]. In this reference accuracy of 20 to $50 \%$ and sensitivity considered as relatively poor are reported for steady state regime $\left(0.03 \mathrm{~K} . \mathrm{W}^{-1} \mathrm{~m}^{2}\right)$. This method suffers from some restrictions:

- very long operative times;

- equilibrium surface temperature reached strongly dependent on convection and radiation heat transfers which take place between the absorbing material and the surroundings. These effects introduce large uncertainties which directly reduce the accuracy of the measurements. The elimination of these parameters in the measuring procedure is a key for the thermographic method enhancement ;

- 3-D conduction effects blurring the images.

\section{Enhancement of the accuracy of the thermographic method}

Most of the problems mentioned above can be solved by avoiding steady-state regime for measurements. The solution consists in producing transient EM fields, creating transient thermal fields to analyze and monitor with IR cameras. Pulsed fields can be used since high energy pulsed EM sources are available. Modulated regime can also be used. One can produce modulation of the field amplitude and detect the thermal effects by lock-in techniques, as currently used in the field of photothermal techniques. In this last field, a method combining the advantages of IR imagery and lock-in detection has been recently proposed [4-5]. We recently demonstrated that such a technique can improve the quantitative characterization of EM phenomena and solve the problems [6-7]. The purpose of this paper is to complete these very first.results.

\section{Reduction by lock-in detection of convective and conductive artefacts}

Let us consider a uniform heat deposition (surface or bulk deposition) with a sinusoidal timevariation of the amplitude, generated by an EM field in an absorbing material, and a radiometric monitoring of the surface temperature. At the beginning, the heating is composed of a slowly 
growing part (very low frequency component) and of a modulated part (at the EM phenomenon frequency). This modulated part is superimposed to a constant heating when the steady-state regime is reached. If the sine variation of the field is used as the reference signal by a lock-in amplifier, it is possible to monitor just the modulated part of the temperature, even during the transient phase. Such a detection leads to the modulus and the phase (with respect to the reference) of the sine temperature signal. A sufficiently high modulation frequency can be chosen to separate the modulated signal from the natural convection frequency domain. In these conditions the analyzed signal will be "convection-free". This can also be demonstrated by looking at the formulae obtained for a simple configuration : the resulting heating of a very thin film, absorbıng a small part of the incident amplitude-modulated EM energy, is proportional to $e^{j \omega t} /(2 h+j \omega C e)$. It is clear that, by increasing the modulation frequency, we can neglect the $h$ term. But, at the same time the heating amplitude decrease. This can be attenuated by increasing the time integration [6], which improves the signal to noise ratio.

Let us consider now a non uniform heating of a thick absorber. Lateral conduction will tend to smooth the distribution details of the surface temperature, which is an undesirable phenomenon. The modulated component of the temperature, at a given point of the surface, will only be influenced by the heating sources within a surrounding volume in the material, defined by $a$ hemisphere of radius $\delta$ which is the diffusion length : $\delta=\sqrt{\kappa / \pi f}$ (figure 1). The influenced domain size can be reduced by increasing $f$ and it is then possible to increase the thermal space resolution of a lock-in radiometric image. In conclusion the lock-in thermographic image will be less influenced by the lateral conduction effect and will have a better resolution.

There is a last interest in lock-in radiometric detection application for heating induced by EM absorption. To visualize the interaction between EM fields and metallic structures, it is possible to coat them with a magnetic or conductive paint. The coat heating can be related to the surface currents. In steady-state regime, the high thermal conductivity of the structures will impede the coat heating to be an accurate print of the surface currents. If the field is amplitude modulated at a sufficiently high frequency to obtain a diffusion length $\delta$ smaller than the coat thickness, then the modulated heating will not be blurred by the thermal diffusion into the metallic substrate (figure 2 ).

\section{Lock-in thermographic system developed at ONERA}

The principle of the lock-in thermography has been presented previously [6]. In the present case we are interested in both modulus image which is proportional to the source intensity (the EM field intensity) and phase image which is only dependent on the heat transfer phenomena.

As illustration of the efficiency of the method, images obtained with a set-up specially arranged for the system validation are given here. The camera ( Agema 782 LW) is viewing an extended blackboby. Two mechanical choppers are placed between the camera and the blackbody, masking it partially. This validation experiment is presented in figure 3 which gives a schematic view (figure $3 a$ ) and the modulus and phase images. The blackbody temperature is $38^{\circ} \mathrm{C}$. The chopper (4) is running at a frequency $(8.3 \mathrm{~Hz}$ ) different from that of the chopper (2) delivering the reference signal for the lock-in thermographic system $(7.85 \mathrm{~Hz})$. Figure $3 b, 3 c$, 3d show that the rejection of the second frequency is as good as the rejection of the continuous background $(0 \mathrm{~Hz})$. The influence of the number of integrated images is clearly shown. The rejection is only good, in the present conditions for 500 integrated images. The quality of the rejection and the necessary number of integrated images is dependent on both the modulation frequency of the observed phenomenon and the camera scanning frequency. In practice, it is necessary to optimize the choice of the modulation frequency, since the scanning frequency is not adjustable.

In figure $3 e, 3 f$, the situation is the same but without the chopper (4), with a modulation frequency of $19,21 \mathrm{~Hz}$ and 2500 integrated images. The system efficiency in eliminating the continuous part of the heating is shown : the directly viewed part of the blackbody is at a zero temperature and the part masked by the chopper is at an uniform temperature (figure $3 e$ : modulus) with iso-phase lines which are radii of the chopper (figure $3 f$ : phase image).

\section{Lock-in correction of the convective exchanges with atmosphere}

The heating amplitude on the sensitive film induced by the incident EM field is perturbed by convective exchanges with surroundings. Figure $A$ shows the heating on a $50 \mu \mathrm{m}$-thick absorbing film when illuminated by a constant EM field (a) and the amplitude modulated one (b). This EM field tomography is obtained with the experimental arrangement shown in figure 4. The profile of these two thermal maps (figure 5), following the vertical diameter, shows how lock-in thermography eradicates the free convection artefacts. The modulated heating is rather axl symmetrical and near the direct EM measurements. On the contrar the continuous heating leads to a wider heating and the upper part is warmer, breaking up the symmetry. 
http://dx.doi.org/10.21611/qirt.1994.043

\section{Field characterization in a wave guide}

Film tomography can be applied to the analysis of the modes present in a wave guide. A method has been developed at ONERA for identification of these modes from the analysis of a field tomography made by an IR camera viewing a sensitive film placed in a normal section of the guide [6-7] (Figure 6). A 1,5 mm-thick dielectric film with submicronic conductive layer as absorbing medium is used.

This particular application is given here since it presents at the same time the three major problems for the solution of which we use the lock-in thermographic system : free convection developing on the film, important conduction effects to the cold metallic walls of the guide, and finally a complex pattern which can be blurred by the lateral conduction effects in the absorber.

Both constant amplitude and amplitude-modulated EM fields were used. Figure 7a shows the film temperature distribution obtained with the constant amplitude field observed by classical thermography, and figures $7 b$ and $7 c$, the amplitude of the modulated component of the temperature (modulus image) at modulation frequencies of $1 \mathrm{~Hz}$ and $10 \mathrm{~Hz}$. It is clear that using a constant amplitude field and the classical thermographic method leads to results which are far from those obtained with amplitude modulation and lock-in thermography. With the lock-in detection, influence of the cold wall is substantially reduced because the two maxima are more contrasted, the shape of the distribution is more complex and the temperature gradients between the two hot spots and the nearest parts of the cold wall are higher.

The phase images (figure $7 d, 7 e$ ) show that higher modulation frequency induces abetter uniformity which corresponds to a decrease of the artefacts (convection, radiation, conduction). They indicate which part of the thermal field is isophase and can be used for the identification.

\section{Conclusion}

A new method for a better observation of EM fields using IR thermography is presented. The consideration of the thermal phenomena occurring in the absorbing media used as field sensors leads to define a new technique which essentially consists :

- to elaborate very thin films with an absorbing (conductive or magnetic) layer,

- to produce a modulation of the amplitude of the microwave field,

- to detect the modulated part of the film heating using a lock-in thermographic system.

The results obtained by this technique seem very promising. Convective heat losses and lateral heat transfer conduction inside the film itself are strongly reduced, allowing a more precise microwave field mapping. Future research is oriented towards film optimization in view to increase sensitivity, use of higher frequency modulations for a better spatial modulation transfer function, extension of the method to coat metallic structures. Furthermore, with the present improvements the IR thermography technique seems well adapted to microwave holography.

\section{Acknowledgments}

Work supported by the the Service Technique des Programmes Aèronatiques (STPA/EG)

\section{REFERENCES}

[1] NORGARD (J.) and al. - Infrared measurement of electromagnetic fields. Quantitative IR Thermography (QIRT 92), ed. Balageas D., Busse G., Carlomagno G.M., Editions Európ. Thermique et Indust. , Paris, 1992

[2] LEVESQUE (P.), DEOM (A.), BALAGEAS (D.) - Non destructive evaluation of absorbing materials using microwave stimulated IR thermography. Ibidem.

[3] METZGER (D.W.) - Quantification of the thermographic mapping of microwave field $\mathrm{PhD}$ Thesis, Univ. of Colorado, 1991

[4 KUO (P.K.) and al. - Parallel thermal wave imaging using a vector lock-in video technique. Photoacustic \& Photothermal Phen., Springer Series in Opt. Sci., vol. 58, pp. 415-418, 1988.

[5] BUSSE (G.) and al. - Lockin vibrothermal inspection of polymer composites. QIRT 92, ed. Balageas D., Busse G., Carlomagno G.M.,Editions Europ. Thermique et Industrie, Paris, 1992.

[6] BALAGEAS (D.), LEVESQUE (P.), DEOM (A.) - Characterization of electromagnetic fiels using a lock-in IR thermography. SPIE Proc. of the 1993 conference "Thermosense XV", vol. 1993, Orlando, FL, April 1993

[7] BALAGEAS (D.) LEVESQUE (P.) - Imagery and quantitative mapping of electromagnetic phenomena using IR thermography. Proc. "Journèes Maxwell". ed. J.F. Eloy, Publ. CEA/CESTA, Bordeaux, France, May 1993 
http://dx.doi.org/10.21611/qirt.1994.043

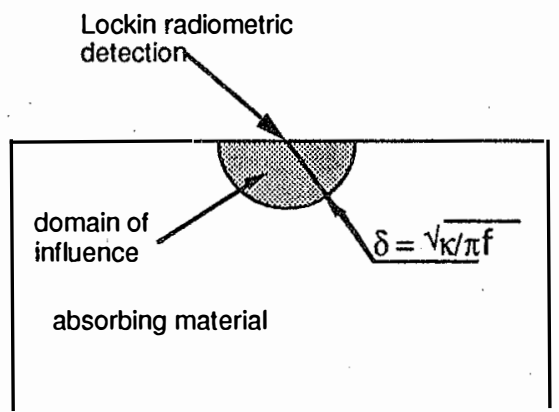

Fig. 1. - Domain of influence for the lock-in radiometric detection. Case of a homogeneous absorbing material

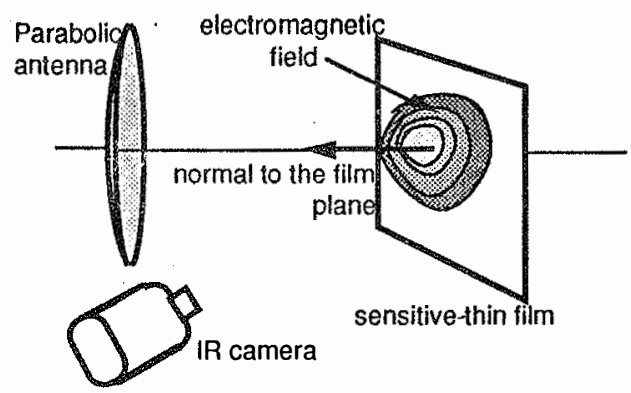

Fig. 4. - Tomography of a field using the sensitive thin film and the IR camera technique. Experimental arrangement.

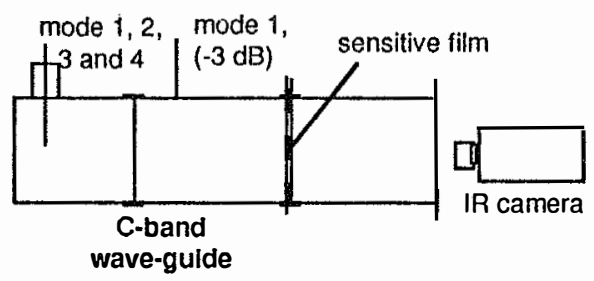

Fig. 6. - Identification of the modes in a wave-guide. Schematic view of the set-up.

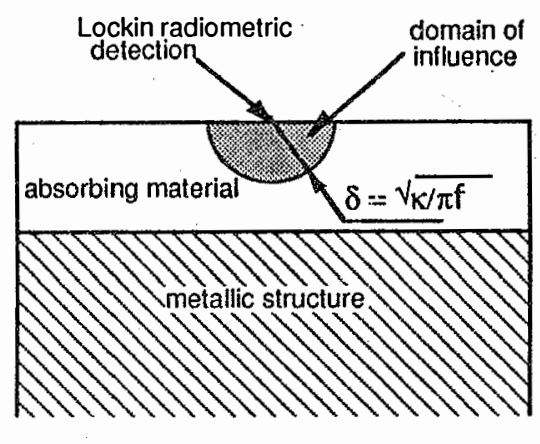

Fig. 2. - Domain of influence for the lock-in radiometric detection. Case of coatings on metallic structure

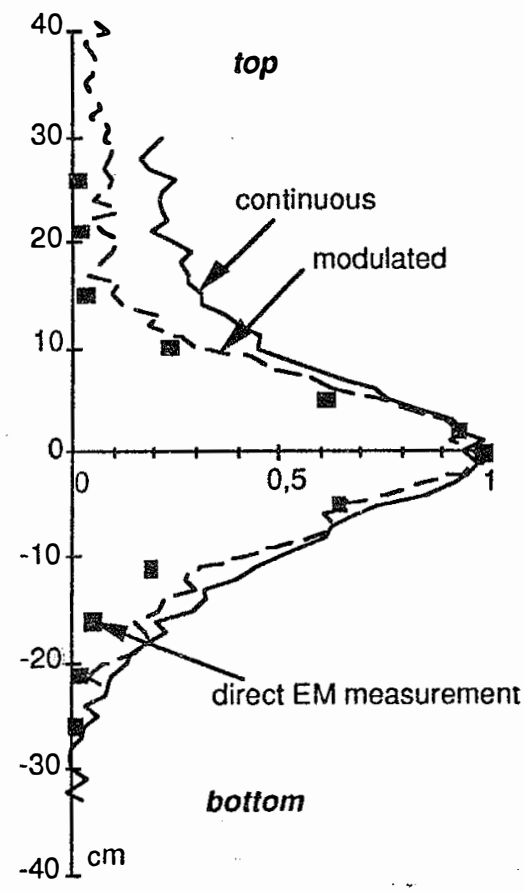

Fig. 5. - Field tomography profiles : eradication of convection effects by lock-in thermography 


\section{http://dx.doi.org/10.21611/qirt.1994.043}
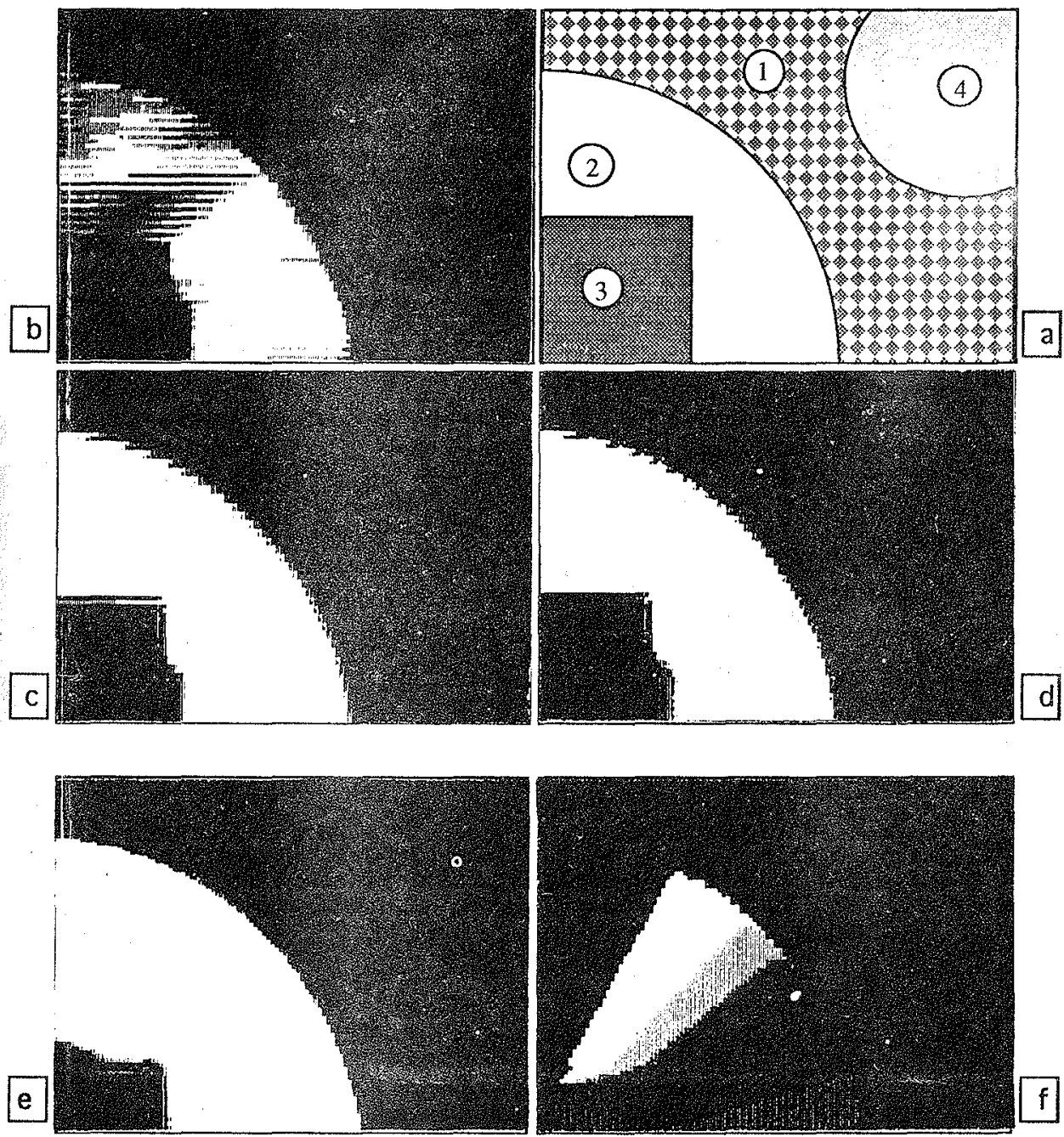

Fig. 3. - Validation experiment of the lock-in IR thermographic system: (a) schematic view with the extended blackbody (1), the chopper disc used as reference for the demodulation, with a modulation frequency of $7.85 \mathrm{~Hz}(2)$, the chopper motor casing (3), a second mechanical chopper whose modulation frequency is $8.3 \mathrm{~Hz}$ (4); (b) modulus image after accumulation of 20 images : - (c) idem after 200 images ; - (d) idem after 500 images ; - (e) modulus after acquisition of 2500 images ( $f=$ $19,21 \mathrm{~Hz}) ;-(f)$ phase after acquisition of 2500 images $(\mathfrak{f}=19,21 \mathrm{~Hz})$ 
http://dx.doi.org/10.21611/qirt.1994.043

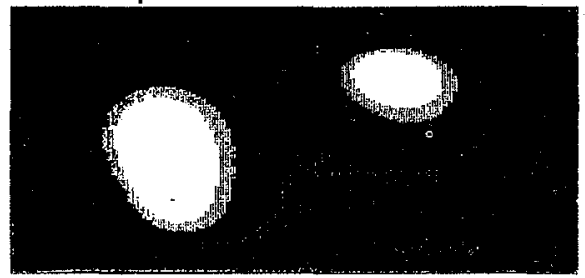

回

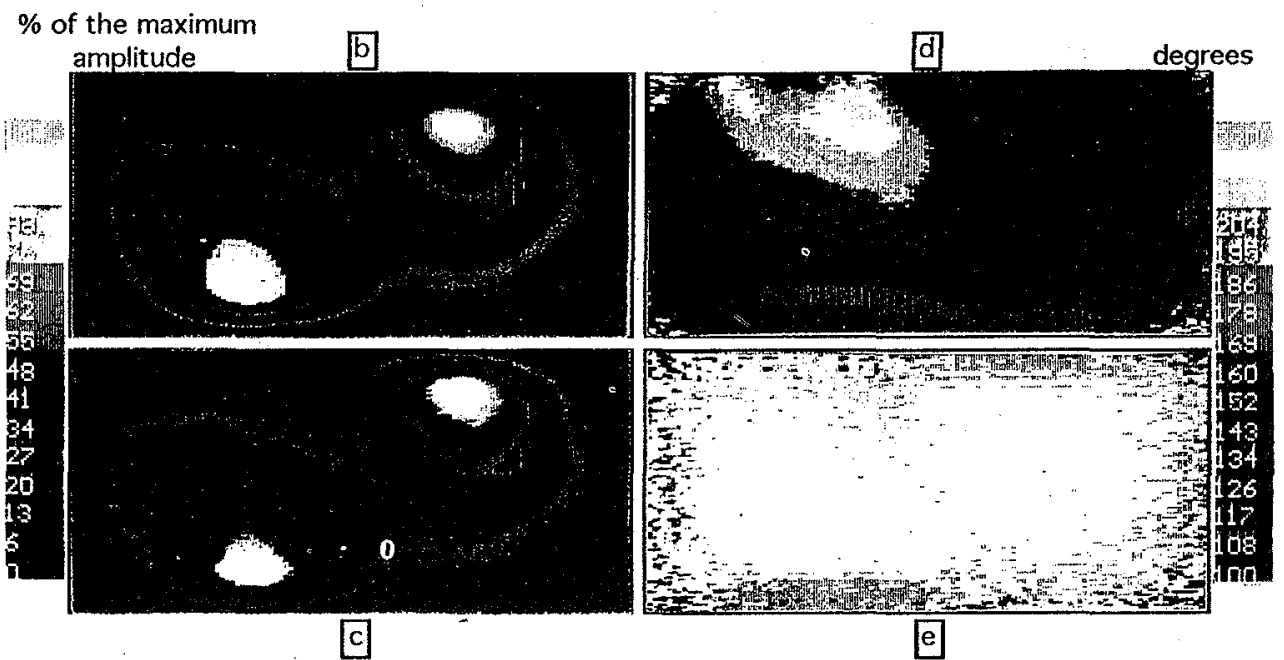

Fig. 7. - Analysis of the energy distribution in the $\mathrm{C}$-band wave-guide, at $8 \mathrm{GHz}$. a) constant amplitude microwave field observed by classical thermography (500 accumulated images).b) c) amplitude-modulated microwave field observed by the lock-in thermographic system, with modulation frequency of $1 \mathrm{~Hz}$ (b) and $10 \mathrm{~Hz}$ (c). The figures present the modulus image after 5000 accumulated images. They are graduated in $\%$ of the maximum value in the field.

d) e) related phase images with modulation frequency of $1 \mathrm{~Hz}(d)$ and $10 \mathrm{~Hz}(e)$, graduated in degrees. 